\title{
LOWER SEMICONTINUITY OF PARAMETRIC GENERALIZED WEAK VECTOR EQUILIBRIUM PROBLEMS
}

\author{
SHENG-JIE LI, HUI-MIN LIU and CHUN-RONG CHEN ${ }^{凶}$
}

(Received 3 March 2009)

\begin{abstract}
In this paper, using a scalarization method, we obtain sufficient conditions for the lower semicontinuity and continuity of the solution mapping to a parametric generalized weak vector equilibrium problem with set-valued mappings.
\end{abstract}

2000 Mathematics subject classification: primary 49K40; secondary 47J20.

Keywords and phrases: lower semicontinuity, solution mappings, parametric generalized weak vector equilibrium problems, scalarization.

\section{Introduction}

It is well known that the vector equilibrium problem provides a unified model of several problems, such as the vector optimization problem, the vector variational inequality problem, the vector complementarity problem and the vector saddle point problem. In the last two decades, a great deal of research has been devoted to finding the existence of solutions to vector equilibrium problems in various versions; see, for example, $[5,10,21]$ and the references therein.

Among many desirable properties of the solution sets to vector equilibrium problems, the stability analysis is of considerable interest. Recently, the semicontinuity, especially the lower semicontinuity, of the solution mappings to parametric vector variational inequalities and parametric vector equilibrium problems has been intensively studied in the literature; example include $[1,2,6-8,11-13,15-$ 20]. Among those papers, we observe that the scalarization technique is one efficient approach to deal with the lower semicontinuity and continuity of solution mappings to parametric vector variational inequalities and parametric vector equilibrium problems. In [8], by using a scalarization method, Cheng and Zhu first obtained a result on

This research was partially supported by the National Natural Science Foundation of China (Grant number: 10871216).

(C) 2009 Australian Mathematical Publishing Association Inc. 0004-9727/2009 \$16.00 
the lower semicontinuity of the solution mapping to a finite-dimensional parametric weak vector variational inequality. By virtue of a density result and scalarization technique, Gong and Yao [12] first discussed the lower semicontinuity of the set of efficient solutions to a parametric vector equilibrium problem with vector-valued mappings. By using the ideas of Cheng and Zhu [8], Gong [11] studied the continuity of the solution mapping to a parametric weak vector equilibrium problem with vector-valued mappings. Very recently, based on a scalarization representation of the solution mapping and a property involving the union of a family of lower semicontinuous set-valued mappings, Chen et al. [7] have established the lower semicontinuity and continuity of the solution mapping to a parametric generalized vector equilibrium problem involving set-valued mappings, and the results obtained include the corresponding versions in $[8,11]$ as special cases.

Motivated by the work reported in $[7,8,11]$, in this paper, we mainly discuss the lower semicontinuity of the solution mapping to a parametric generalized weak vector equilibrium problem (PGWVEP) involving set-valued mappings. By using the ideas of Chen et al. [7], we analyze the problem (PGWVEP) with a scalarization method and derive sufficient conditions for the lower semicontinuity and continuity of the solution mapping to (PGWVEP). Our conclusions are new and also include the corresponding results in $[8,11]$ as special cases.

The rest of the paper is organized as follows. In Section 2, we present the problem (PGWVEP), and recall some concepts of semicontinuity and their some properties. In Section 3, we discuss the existence, the lower semicontinuity and continuity of the solution mapping to (PGWVEP), and give examples to illustrate our results.

\section{Preliminaries}

Throughout this paper, let $X$ and $Y$ be real Hausdorff topological vector spaces, and let $Z$ be a real topological space. Assume that $C$ is a pointed closed convex cone in $Y$ with its interior int $C \neq \emptyset$. Let $Y^{*}$ be the topological dual space of $Y$ and let $C^{*}:=\left\{f \in Y^{*} \mid f(y) \geq 0\right.$, for all $\left.y \in C\right\}$ be the dual cone of $C$.

Suppose that $A$ is a nonempty subset of $X$ and $F: A \times A \rightarrow 2^{Y} \backslash\{\emptyset\}$ is a set-valued mapping. We consider the following generalized weak vector equilibrium problem (GWVEP) of finding $x \in A$ such that

$$
F(x, y) \cap(Y \backslash-\operatorname{int} C) \neq \emptyset \quad \forall y \in A .
$$

Note that the existence of solutions to this problem has been investigated in [22] and the references therein.

When the set $A$ and the mapping $F$ are perturbed by a parameter $\mu$ which varies over a set $\Lambda$ of $Z$, we consider the following (PGWVEP) of finding $x \in A(\mu)$ such that

$$
F(x, y, \mu) \cap(Y \backslash-\text { int } C) \neq \emptyset \quad \forall y \in A(\mu),
$$

where $A: \Lambda \rightarrow 2^{X} \backslash\{\emptyset\}$ is a set-valued mapping, $F: B \times B \times \Lambda \subset X \times X \times Z \rightarrow$ $2^{Y} \backslash\{\emptyset\}$ is a set-valued mapping with $A(\Lambda)=\bigcup_{\mu \in \Lambda} A(\mu) \subset B$. 
For each $\mu \in \Lambda$, let $S_{w}(\mu)$ denote the solution set of (PGWVEP), namely,

$$
S_{w}(\mu)=\{x \in A(\mu) \mid F(x, y, \mu) \cap(Y \backslash-\text { int } C) \neq \emptyset, \quad \forall y \in A(\mu)\} .
$$

In this paper, by applying a scalarization method used in [7], we discuss the lower semicontinuity and continuity of $S_{w}(\cdot)$ as a set-valued mapping from the set $\Lambda$ into $X$.

Suppose that $\Lambda$ and $\Omega$ are Hausdorff topological spaces and $G: \Lambda \rightarrow 2^{\Omega}$ is a setvalued mapping with nonempty values.

\section{DEFINITION 2.1 (Aubin and Ekeland [3]).}

(i) $G$ is lower semicontinuous (1.s.c.) at $\bar{\lambda} \in \Lambda$ if for any open set $Q \subset \Omega$ with $G(\bar{\lambda}) \cap Q \neq \emptyset$, there exists a neighborhood $N(\bar{\lambda})$ of $\bar{\lambda}$ such that $G(\lambda) \cap Q \neq \emptyset$, for all $\lambda \in N(\bar{\lambda})$.

(ii) $G$ is upper semicontinuous (u.s.c.) at $\bar{\lambda}$ if for any open set $Q \subset \Omega$ with $G(\bar{\lambda}) \subset Q$, there exists a neighborhood $N(\bar{\lambda})$ of $\bar{\lambda}$ such that $G(\lambda) \subset Q$, for all $\lambda \in N(\bar{\lambda})$.

(iii) $G$ is closed at $\bar{\lambda}$ if for each net $\left(\lambda_{\alpha}, x_{\alpha}\right) \in \operatorname{graph}(G):=\{(\lambda, x) \mid \lambda \in \Lambda, x \in$ $G(\lambda)\}$ with $\left(\lambda_{\alpha}, x_{\alpha}\right) \rightarrow(\bar{\lambda}, \bar{x})$, it yields $(\bar{\lambda}, \bar{x}) \in \operatorname{graph}(G)$.

We say that $G$ is 1.s.c. (respectively u.s.c., closed) on $\Lambda$, if it is 1.s.c. (respectively u.s.c., closed) at each $\lambda \in \Lambda$. Here $G$ is said to be continuous on $\Lambda$ if it is both 1.s.c. and u.s.c. on $\Lambda$.

REMARK 2.2.

(i) In [7], Chen et al. have established the lower semicontinuity and continuity of the solution mapping to the following parametric generalized vector equilibrium problem (PGVEP) of finding $x \in A(\mu)$ such that

$$
F(x, y, \mu) \subset Y \backslash-\operatorname{int} C \quad \forall y \in A(\mu) .
$$

Obviously, the solution set $S(\mu)$ of (PGVEP) is included in the solution set $S_{w}(\mu)$ of (PGWVEP). Example 4.1 of [1] indicates that when $S_{w}(\cdot)$ is 1.s.c., $S(\cdot)$ is not 1.s.c., while [1, Example 4.2] indicates that when $S(\cdot)$ is 1.s.c., $S_{w}(\cdot)$ is not 1.s.c. Hence, it is necessary to investigate the lower semicontinuity of $S_{w}(\cdot)$ to (PGWVEP).

(ii) When $F$ is a vector-valued mapping, the models (PGWVEP) and (PGVEP) simultaneously reduce to the parametric vector equilibrium problem (PVEP) of finding $x \in A(\mu)$ such that

$$
F(x, y, \mu) \notin-\operatorname{int} C \quad \forall y \in A(\mu) .
$$

The results on the lower semicontinuity of the solution mappings to the special cases of (PVEP) have been obtained in [8, 11]. The scalarization method used in [7] to deal with the lower semicontinuity of solution mappings is different from those used in $[8,11]$, and is also different from other methods used in the literature, such as $[1,2,6,13,17,18]$. 
Proposition 2.3 (Ferro [9]; Aubin and Ekeland [3]).

(i) $G$ is l.s.c. at $\bar{\lambda}$ if and only iffor any net $\left\{\lambda_{\alpha}\right\} \subset \Lambda$ with $\lambda_{\alpha} \rightarrow \bar{\lambda}$ and any $\bar{x} \in G(\bar{\lambda})$, there exists $x_{\alpha} \in G\left(\lambda_{\alpha}\right)$ such that $x_{\alpha} \rightarrow \bar{x}$.

(ii) If $G$ has compact values $(G(\lambda)$ is a compact set for each $\lambda \in \Lambda)$, then $G$ is u.s.c. at $\bar{\lambda}$ if and only if for any net $\left\{\lambda_{\alpha}\right\} \subset \Lambda$ with $\lambda_{\alpha} \rightarrow \bar{\lambda}$ and for any $x_{\alpha} \in G\left(\lambda_{\alpha}\right)$, there exist $\bar{x} \in G(\bar{\lambda})$ and a subnet $\left\{x_{\beta}\right\}$ of $\left\{x_{\alpha}\right\}$, such that $x_{\beta} \rightarrow \bar{x}$.

The following lemma plays an important role in the proof of the lower semicontinuity of the solution mapping $S_{w}(\cdot)$.

LEMma 2.4 (Berge [4, Theorem 2, p. 114]). The union $\Gamma=\bigcup_{i \in I} \Gamma_{i}$ of a family of l.s.c. set-valued mappings $\Gamma_{i}$ from a topological space $X$ into a topological space $Y$ is also an l.s.c. set-valued mapping from $X$ into $Y$, where $I$ is an index set.

\section{Lower semicontinuity and continuity}

For each $f \in C^{*} \backslash\{0\}$ and for each $\mu \in \Lambda$, let $\bar{S}_{f}(\mu)$ denote the set of $f$-efficient solutions to (PGWVEP), that is,

$$
\bar{S}_{f}(\mu)=\{x \in A(\mu) \mid \forall y \in A(\mu), \exists z \in F(x, y, \mu), \text { such that } f(z) \geq 0\} ;
$$

and let $\bar{V}_{f}$ denote the set of $f$-efficient solutions to (GWVEP), namely,

$$
\bar{V}_{f}=\{x \in A \mid \forall y \in A, \exists z \in F(x, y), \text { such that } f(z) \geq 0\} .
$$

Before discussing the lower semicontinuity of the solution mapping $S_{w}(\cdot)$, we first give a kind of sufficient conditions which ensure that $\bar{S}_{f}(\mu) \neq \emptyset$ for all $f \in C^{*} \backslash\{0\}$ and $\mu \in \Lambda$. For the sake of simplicity of presentation, we first give the sufficient condition which ensures the existence of solutions of $\bar{S}_{f}$ without involving the parameter $\mu$. Now we recall some concepts.

A set-valued mapping $E: A \rightarrow 2^{A}$ is called a KKM mapping if $\operatorname{co}\left\{x_{1}, \ldots, x_{n}\right\} \subset$ $\bigcup_{i=1}^{n} E\left(x_{i}\right)$ for any finite subset $\left\{x_{1}, \ldots, x_{n}\right\}$ of $A$, where $\operatorname{co}(D)$ denotes the convex hull of the set $D$.

The set-valued mapping $G: A \rightarrow 2^{Y}$ is said to be $C$-convex on $A$ if for any $x_{1}, x_{2} \in A$ and $\lambda \in[0,1], \lambda G\left(x_{1}\right)+(1-\lambda) G\left(x_{2}\right) \subset G\left(\lambda x_{1}+(1-\lambda) x_{2}\right)+C$.

PROPOSITION 3.1. Suppose that the following conditions are satisfied:

(i) A is a nonempty compact convex set;

(ii) for each $y \in A, F(\cdot, y)$ is u.s.c. with nonempty compact values on $A$;

(iii) $F(x, x) \subset C$, for all $x \in A$, and $F(x, \cdot)$ is $C$-convex on $A$.

Then, for each $f \in C^{*} \backslash\{0\}, \bar{V}_{f} \neq \emptyset$.

Proof. Define $M: A \rightarrow 2^{A}$ by

$$
M(y)=\{x \in A \mid \exists z \in F(x, y), \text { such that } f(z) \geq 0\} \quad \forall y \in A .
$$


We first prove that for any $y \in A, M(y)$ is a closed set. Let $x_{\alpha} \in M(y)$ and $x_{\alpha} \rightarrow x_{0}$. Then $x_{0} \in A$ since $A$ is compact. It follows from $x_{\alpha} \in M(y)$ that there exists $z_{\alpha} \in F\left(x_{\alpha}, y\right)$ such that

$$
f\left(z_{\alpha}\right) \geq 0 .
$$

Since $F(\cdot, y)$ is u.s.c. at $x_{0}$ with compact values, there exists $z_{0} \in F\left(x_{0}, y\right)$ such that $z_{\alpha} \rightarrow z_{0}$ (taking a subnet if necessary). It follows from the continuity of $f$ and (3.1) that $f\left(z_{0}\right) \geq 0$. Thus, $x_{0} \in M(y)$. So $M(y)$ is a closed set. Moreover, since $M(y) \subset A$ and $A$ is compact, $M(y)$ is also a compact set.

Next, we show that $M$ is a KKM mapping. Suppose that this is false. Then there exist a finite subset $\left\{y_{1}, \ldots, y_{n}\right\} \subset A$ and $t_{1}, \ldots, t_{n} \geq 0$ with $\sum_{i=1}^{n} t_{i}=1$ such that $\bar{z}=\sum_{i=1}^{n} t_{i} y_{i} \notin \bigcup_{i=1}^{n} M\left(y_{i}\right)$. Then, for each $i \in\{1,2, \ldots, n\}, \bar{z} \notin M\left(y_{i}\right)$, that is, for all $z_{i} \in F\left(\bar{z}, y_{i}\right), f\left(z_{i}\right)<0$. Without loss of generality, we can choose $\left\{z_{1}, \ldots, z_{n}\right\}$ such that for $j=1,2, \ldots, n, z_{j} \in F\left(\bar{z}, y_{j}\right)$ and

$$
f\left(z_{j}\right)<0
$$

From condition (iii), we have

$$
\sum_{j=1}^{n} t_{j} z_{j} \in \sum_{j=1}^{n} t_{j} F\left(\bar{z}, y_{j}\right) \subset F(\bar{z}, \bar{z})+C \subset C+C \subset C .
$$

By the linearity of $f$ and $f \in C^{*} \backslash\{0\}$, we obtain

$$
\sum_{j=1}^{n} t_{j} f\left(z_{j}\right)=f\left(\sum_{j=1}^{n} t_{j} z_{j}\right) \geq 0 .
$$

On the other hand, it follows from $t_{j} \geq 0, j=1,2, \ldots, n$, with $\sum_{j=1}^{n} t_{j}=1$ and (3.2) that

$$
\sum_{j=1}^{n} t_{j} f\left(z_{j}\right)<0
$$

a contradiction. Hence, $M$ is a KKM mapping.

By the well-known Ky Fan lemma (see, for example, [13, Lemma 2.2]), we have $\bigcap_{y \in A} M(y) \neq \emptyset$. Thus, $\bar{V}_{f}=\bigcap_{y \in A} M(y) \neq \emptyset$.

From Proposition 3.1, we have the following existence result readily.

Proposition 3.2. For each $\mu \in \Lambda$, suppose that the following conditions are satisfied:

(i) $A(\mu)$ is a nonempty compact convex set;

(ii) for each $y \in A(\mu), F(\cdot, y, \mu)$ is u.s.c. with nonempty compact values on $A(\mu)$;

(iii) $F(x, x, \mu) \subset C$, for all $x \in A(\mu)$, and $F(x, \cdot, \mu)$ is $C$-convex on $A(\mu)$.

Then, for each $f \in C^{*} \backslash\{0\}$ and $\mu \in \Lambda, \bar{S}_{f}(\mu) \neq \emptyset$. 
Now we establish the lower semicontinuity and continuity of $S_{w}(\cdot)$ to (PGWVEP). In what follows, we assume that $\bar{S}_{f}(\mu) \neq \emptyset$ for all $f \in C^{*} \backslash\{0\}$ and $\mu \in \Lambda$.

LEMMA 3.3. For each $\mu \in \Lambda$, suppose that for each $x \in S_{w}(\mu)$, and for all $y \in A(\mu)$, there exists a selection $z(y)$ of $F(x, y, \mu) \backslash-\operatorname{int} C$ (that is, $z(y) \in F(x, y, \mu) \backslash$ $-\operatorname{int} C)$, such that $\bigcup_{y \in A(\mu)} z(y)+C$ is a convex set. Then

$$
S_{w}(\mu)=\bigcup_{f \in C^{*} \backslash\{0\}} \bar{S}_{f}(\mu) .
$$

PRoOF. ' $\supset$ ' Let $x \in \bigcup_{f \in C^{*} \backslash\{0\}} \bar{S}_{f}(\mu)$. Then there exists $f^{\prime} \in C^{*} \backslash\{0\}$ such that $x \in \bar{S}_{f^{\prime}}(\mu)$. Thus, $x \in A(\mu)$ and for all $y \in A(\mu)$, there exists $z \in F(x, y, \mu)$ such that $f^{\prime}(z) \geq 0$. We deduce that $z \notin-$ int $C$. Otherwise, if $z \in-$ int $C$, then it follows from $f^{\prime} \in C^{*} \backslash\{0\}$ that $f^{\prime}(z)<0$, which yields a contradiction. Hence, we obtain that there exists $z \in F(x, y, \mu) \cap(Y \backslash-$ int $C)$ for all $y \in A(\mu)$, which shows that $x \in S_{w}(\mu)$.

' $\subset$ ' Let $x \in S_{w}(\mu)$. Then, for every $y \in A(\mu)$, there exists a selection $z(y)$ of $F(x, y, \mu) \backslash-$ int $C$, such that $\bigcup_{y \in A(\mu)} z(y)+C$ is a convex set. We have

$$
\left(\bigcup_{y \in A(\mu)} z(y)\right) \cap(-\operatorname{int} C)=\emptyset,
$$

and, hence,

$$
\left(\bigcup_{y \in A(\mu)} z(y)+C\right) \cap(-\operatorname{int} C)=\emptyset .
$$

As $\bigcup_{y \in A(\mu)} z(y)+C$ is convex, by the well-known Eidelheit's separation theorem (see [14, Theorem 3.16]), there exist a continuous linear functional $f \in Y^{*} \backslash\{0\}$ and a real number $\gamma$ such that

$$
f(\hat{c})<\gamma \leq f(z+c),
$$

for all $z \in \bigcup_{y \in A(\mu)} z(y), c \in C$ and $\hat{c} \in-\operatorname{int} C$. Since $C$ is a cone, we obtain $f(\hat{c}) \leq 0$ for all $\hat{c} \in-\operatorname{int} C$. Thus, $f(\hat{c}) \geq 0$ for all $\hat{c} \in C$, that is $f \in C^{*}$. Moreover, it follows from $c \in C, \hat{c} \in-$ int $C$ and the continuity of $f$ that $f(z) \geq 0$ for all $z \in \bigcup_{y \in A(\mu)} z(y)$. Thus, for all $y \in A(\mu)$, there exists $z(y) \in F(x, y, \mu)$, such that $f(z(y)) \geq 0$. Hence, we obtain $x \in \bar{S}_{f}(\mu) \subset \bigcup_{f \in C^{*} \backslash\{0\}} \bar{S}_{f}(\mu)$.

REMARK 3.4. When $F$ is a vector-valued mapping, the assumption condition of Lemma 3.3 automatically reduces to that for each $x \in S_{w}(\mu), F(x, A(\mu), \mu)+$ $C=\bigcup_{y \in A(\mu)} F(x, y, \mu)+C$ is a convex set. Clearly, in order to avoid any information on the solution set $S_{w}(\mu)$, we can strengthen it to that for each $x \in A(\mu)$, $F(x, A(\mu), \mu)+C$ is a convex set. Thus, Lemma 3.3 reduces to [11, Theorem 2.1].

REMARK 3.5. In [7, Lemma 3.1], in order to obtain a similar scalarization representation of the solution mapping to (PGVEP), the authors suppose that for each $x \in A(\mu), F(x, A(\mu), \mu)+C=\bigcup_{y \in A(\mu)} F(x, y, \mu)+C$ is a convex set, where $F$ is 
a set-valued mapping. Now we give an example to show that this convexity assumption is different from the convexity assumption used in Lemma 3.3 of this paper.

EXAmple 3.6. Let $X=Z=R, Y=R^{2}, \Lambda=(0,1) \subset R$ and $C=R_{+}^{2}:=[0,+\infty) \times$ $[0,+\infty)$. For each $\mu \in(0,1)$, let $A(\mu)=[0,1]$. For each $\mu \in \Lambda$ and $x \in A(\mu)$, for all $y \in A(\mu)$, let

$$
G(x, y, \mu)=\{\mu(-2,2),(0,-x-y)\}
$$

and

$$
\begin{gathered}
F(x, y, \mu)=\left\{(a, b) \in R^{2} \mid(a, b)=t(c, d)+(1-t)(1,0),\right. \\
t \in[0,1],(c, d) \in G(x, y, \mu)\} .
\end{gathered}
$$

Then, for each $x \in A(\mu)$, obviously, $F(x, A(\mu), \mu)+R_{+}^{2}$ is not a convex set. However, for every $y \in A(\mu)$, letting $z(y)=(0,-x-y)$, we have $z(y) \in$ $F(x, y, \mu) \backslash$-int $R_{+}^{2}$. It is clear that $\bigcup_{y \in A(\mu)} z(y)+R_{+}^{2}$ is a convex set.

Now we state our main result as follows.

THEOREM 3.7. Suppose that the following conditions are satisfied:

(i) $A$ is continuous with nonempty compact values on $\Lambda$;

(ii) $F$ is u.s.c. with nonempty compact values on $B \times B \times \Lambda$;

(iii) $F(\cdot, \cdot, \mu)$ is $C$-strictly monotone on $A(\mu) \times A(\mu)$ for any given $\mu \in \Lambda$, that is, for all $x, y \in A(\mu)$ and $x \neq y, F(x, y, \mu)+F(y, x, \mu) \subset-\operatorname{int} C$;

(iv) for each $\mu \in \Lambda$, for each $x \in S_{w}(\mu)$, and for all $y \in A(\mu)$, there exists a selection $z(y)$ of $F(x, y, \mu) \backslash-$ int $C$, such that $\bigcup_{y \in A(\mu)} z(y)+C$ is a convex set.

Then, $S_{w}(\cdot)$ is l.s.c. on $\Lambda$.

We remark that the $C$-strict monotonicity of $F$ in Theorem 3.7 is essential. Example 3.1 of [7] illustrates this fact, since $F$ is assumed to be single-valued therein.

PROOF. We now prove that for each $f \in C^{*} \backslash\{0\}, \bar{S}_{f}(\cdot)$ is 1.s.c. on $\Lambda$.

Suppose to the contrary that there exist $f \in C^{*} \backslash\{0\}$ and $\mu_{0} \in \Lambda$ such that $\bar{S}_{f}(\cdot)$ is not 1.s.c. at $\mu_{0}$. Then there exist $\left\{\mu_{\alpha}\right\}$ with $\mu_{\alpha} \rightarrow \mu_{0}$ and $x_{0} \in \bar{S}_{f}\left(\mu_{0}\right)$, such that for any $x_{\alpha} \in \bar{S}_{f}\left(\mu_{\alpha}\right), x_{\alpha} \nrightarrow x_{0}$.

Since $A(\cdot)$ is 1.s.c. at $\mu_{0}$, there exists a net $\bar{x}_{\alpha} \in A\left(\mu_{\alpha}\right)$ such that $\bar{x}_{\alpha} \rightarrow x_{0}$. For any $y_{\alpha} \in \bar{S}_{f}\left(\mu_{\alpha}\right)$, because $A(\cdot)$ is u.s.c. at $\mu_{0}$ with compact values, there exist $y_{0} \in A\left(\mu_{0}\right)$ and a subnet $\left\{y_{\beta}\right\}$ of $\left\{y_{\alpha}\right\}$ such that $y_{\beta} \rightarrow y_{0}$. It follows from $x_{0} \in \bar{S}_{f}\left(\mu_{0}\right)$ and $y_{0} \in A\left(\mu_{0}\right)$ that there exists $z_{0 x} \in F\left(x_{0}, y_{0}, \mu_{0}\right)$ such that

$$
f\left(z_{0 x}\right) \geq 0 \text {. }
$$

On the other hand, since $y_{\beta} \in \bar{S}_{f}\left(\mu_{\beta}\right)$ and $\bar{x}_{\beta} \in A\left(\mu_{\beta}\right)$, there exists $z_{\beta} \in$ $F\left(y_{\beta}, \bar{x}_{\beta}, \mu_{\beta}\right)$ such that

$$
f(z \beta) \geq 0 \text {. }
$$


Because $F(\cdot, \cdot, \cdot)$ is u.s.c. at $\left(y_{0}, x_{0}, \mu_{0}\right)$ with compact values, there exists $z_{0 y} \in$ $F\left(y_{0}, x_{0}, \mu_{0}\right)$ such that $z_{\beta} \rightarrow z_{0 y}$ (taking a subnet if necessary). It follows from the continuity of $f$ and (3.4) that

$$
f\left(z_{0 y}\right) \geq 0 .
$$

By (3.3), (3.5) and the linearity of $f$, we obtain

$$
f\left(z_{0 x}+z_{0 y}\right)=f\left(z_{0 x}\right)+f\left(z_{0 y}\right) \geq 0 .
$$

Assume that $y_{0} \neq x_{0}$. Since $F\left(\cdot, \cdot, \mu_{0}\right)$ is $C$-strictly monotone on $A\left(\mu_{0}\right) \times A\left(\mu_{0}\right)$, we have

$$
F\left(x_{0}, y_{0}, \mu_{0}\right)+F\left(y_{0}, x_{0}, \mu_{0}\right) \subset-\text { int } C .
$$

Then it follows from $f \in C^{*} \backslash\{0\}$ and $z_{0 x}+z_{0 y} \in-$ int $C$ that

$$
f\left(z_{0 x}+z_{0 y}\right)<0,
$$

which contradicts (3.6). Therefore, $y_{0}=x_{0}$. This contradicts the assumption that $y_{0} \neq x_{0}$.

By virtue of condition (iv) and Lemma 3.3, for each $\mu \in \Lambda$, it holds that

$$
S_{w}(\mu)=\bigcup_{f \in C^{*} \backslash\{0\}} \bar{S}_{f}(\mu) .
$$

Since for each $f \in C^{*} \backslash\{0\}, \bar{S}_{f}(\cdot)$ is 1.s.c. on $\Lambda$, in view of Lemma 2.4, we obtain that $S_{w}(\cdot)$ is 1.s.c. on $\Lambda$.

Furthermore, we point out that under the assumptions of Theorem 3.7, the solution mapping $S_{w}(\cdot)$ is both continuous and closed. Thus, Theorem 3.7 is essentially a sufficient condition for the continuity of the solution mapping $S_{w}(\cdot)$.

THEOREM 3.8. Suppose that all conditions of Theorem 3.7 are satisfied. Then, $S_{w}(\cdot)$ is both continuous and closed on $\Lambda$.

PROOF. In fact, the upper semicontinuity and closedness of $S_{w}(\cdot)$ could be directly implied by [1, Theorem 3.2], without using the $C$-strict monotonicity and the convexity assumptions.

Motivated by [11, Example 4.1], we give the following example to illustrate Theorem 3.8.

EXAmple 3.9. Let $X=Y=R^{2}, Z=R, C=R_{+}^{2}$ and $\Lambda=(0,1) \subset R$. For any given $\mu \in(0,1)$, let

$$
A(\mu)=\left\{x=\left(x_{1}, x_{2}\right) \in R^{2} \mid x_{1}^{2}+x_{2}^{2} \leq \mu^{2}\right\} .
$$

Note that $A(\mu) \subset\left\{x=\left(x_{1}, x_{2}\right) \in R^{2} \mid x_{1}^{2}+x_{2}^{2} \leq 1\right\}:=B$, for all $\mu \in \Lambda$.

Let

$$
F_{1}(x)=\left(\alpha x_{1}, x_{2}\right), \quad F_{2}(x)=\left(x_{1}, x_{2}\right) \quad \forall x=\left(x_{1}, x_{2}\right) \in R^{2},
$$


where the constant $\alpha>0$ is fixed. Define the mappings $\varphi: B \times B \times \Lambda \rightarrow R^{2}$ and $\psi: B \times \Lambda \rightarrow R^{2}$ by

$$
\begin{gathered}
\varphi(x, y, \mu)=\left(\left\langle\mu F_{1}(x), y-x\right\rangle,\left\langle\mu F_{2}(x), y-x\right\rangle\right) \quad \forall x, y \in B, \mu \in \Lambda, \\
\psi(x, \mu)=\mu\left(x_{1}, x_{2}^{2}\right) \quad \forall x=\left(x_{1}, x_{2}\right) \in B, \mu \in \Lambda .
\end{gathered}
$$

For $x=\left(x_{1}, x_{2}\right), y=\left(y_{1}, y_{2}\right) \in A(\mu)$, let

$$
F_{v}(x, y, \mu)=\varphi(x, y, \mu)+\psi(y, \mu)-\psi(x, \mu):=(a, b),
$$

and

$$
F(x, y, \mu)=\left\{(u, v) \in R^{2} \mid(u, v)=t(a, b)+(1-t)(-2 \alpha-4,-5), t \in[0,1]\right\},
$$

where $F_{v}: B \times B \times \Lambda \rightarrow R^{2}, F: B \times B \times \Lambda \rightarrow 2^{R^{2}}$ and

$$
(a, b)=\mu\left(\alpha x_{1} y_{1}-\alpha x_{1}^{2}+x_{2} y_{2}-x_{2}^{2}+y_{1}-x_{1}, x_{1} y_{1}-x_{1}^{2}+x_{2} y_{2}-2 x_{2}^{2}+y_{2}^{2}\right) .
$$

For all $\mu \in \Lambda$ and $x, y \in A(\mu)$, we have $-2 \alpha-4<a$, and $-5<b$. So, $F(x, y, \mu) \subset$ $(a, b)-R_{+}^{2}$.

It follows from [11, Example 4.1] that for each

$$
f \in C^{*} \backslash\{0\}, V_{f}(\mu)=\left\{x \in A(\mu) \mid f\left(F_{v}(x, y, \mu)\right) \geq 0 \forall y \in A(\mu)\right\} \neq \emptyset .
$$

Obviously, $V_{f}(\mu) \subset \bar{S}_{f}(\mu)$. So, for each $f \in C^{*} \backslash\{0\}, \bar{S}_{f}(\mu) \neq \emptyset$.

It is clear that the conditions (i) and (ii) in Theorem 3.7 are satisfied. Now we check the condition (iii). Clearly,

$$
F(y, x, \mu)=\left\{(u, v) \in R^{2} \mid(u, v)=t(c, d)+(1-t)(-2 \alpha-4,-5), t \in[0,1]\right\},
$$

where

$$
(c, d)=\mu\left(\alpha x_{1} y_{1}-\alpha y_{1}^{2}+x_{2} y_{2}-y_{2}^{2}+x_{1}-y_{1}, x_{1} y_{1}-y_{1}^{2}+x_{2} y_{2}-2 y_{2}^{2}+x_{2}^{2}\right) .
$$

We also have $F(y, x, \mu) \subset(c, d)-R_{+}^{2}$. Thus, for each $\mu \in \Lambda$, for any $x, y \in A(\mu)$ and $x \neq y$,

$$
\begin{aligned}
F(x, y, \mu)+F(y, x, \mu) \subset & (a, b)-R_{+}^{2}+(c, d)-R_{+}^{2} \\
= & \mu\left(2 \alpha x_{1} y_{1}-\alpha y_{1}^{2}-\alpha x_{1}^{2}+2 x_{2} y_{2}-y_{2}^{2}-x_{2}^{2},\right. \\
& \left.\quad 2 x_{1} y_{1}-x_{1}^{2}-y_{1}^{2}+2 x_{2} y_{2}-y_{2}^{2}-x_{2}^{2}\right)-R_{+}^{2} \\
= & \mu\left(-\alpha\left(x_{1}-y_{1}\right)^{2}-\left(x_{2}-y_{2}\right)^{2},\right. \\
& \left.\quad-\left(x_{1}-y_{1}\right)^{2}-\left(x_{2}-y_{2}\right)^{2}\right)-R_{+}^{2} \\
& \subset-\operatorname{int} R_{+}^{2}-R_{+}^{2} \\
= & - \text { int } R_{+}^{2} .
\end{aligned}
$$

Hence, condition (iii) of Theorem 3.7 holds. 
Since for each $x \in S_{w}(\mu)$, we have for all $y \in A(\mu)$, there is an $z_{y} \in F(x, y, \mu) \backslash$ -int $R_{+}^{2}$, hence it is clear that $\left(a_{y}, b_{y}\right) \in F(x, y, \mu) \backslash$-int $R_{+}^{2}$, where $\left(a_{y}, b_{y}\right)$ means that $(a, b)$ depends on $y$. Without loss of generality, set $z(y)=\left(a_{y}, b_{y}\right)$. Thus, we obtain $\bigcup_{y \in A(\mu)} z(y)=F_{v}(x, A(\mu), \mu)$. It follows from [11, Example 4.1] that $\bigcup_{y \in A(\mu)} z(y)+C=F_{v}(x, A(\mu), \mu)+C$ is a convex set. Thus, condition (iv) of Theorem 3.7 is also satisfied.

So, all of the conditions of Theorem 3.7 hold. By Theorem 3.8, $S_{w}(\cdot)$ is continuous and closed on $\Lambda$.

When the mapping $F$ is vector-valued, we obtain the following corollary.

Corollary 3.10. Suppose that $F$ is a vector-valued mapping and the following conditions are satisfied:

(i) $A$ is continuous with nonempty compact values on $\Lambda$;

(ii) $F$ is continuous on $B \times B \times \Lambda$;

(iii) $F(\cdot, \cdot, \mu)$ is $C$-strictly monotone on $A(\mu) \times A(\mu)$ for any given $\mu \in \Lambda$, that is, for all $x, y \in A(\mu)$ and $x \neq y, F(x, y, \mu)+F(y, x, \mu) \in-\operatorname{int} C$;

(iv) for each $\mu \in \Lambda$ and for each $x \in A(\mu), F(x, \cdot, \mu)$ is $C$-convexlike on $A(\mu)$, that is, for any $x_{1}, x_{2} \in A(\mu)$ and any $\rho \in[0,1]$, there exists $x_{3} \in A(\mu)$ such that $\rho F\left(x, x_{1}, \mu\right)+(1-\rho) F\left(x, x_{2}, \mu\right) \in F\left(x, x_{3}, \mu\right)+C$.

Then, $S_{w}(\cdot)$ is both continuous and closed on $\Lambda$.

Proof. For each $\mu \in \Lambda$ and for each $x \in A(\mu)$, since $F(x, \cdot, \mu)$ is $C$-convexlike on $A(\mu), F(x, A(\mu), \mu)+C$ is a convex set. Then it follows from Remark 3.4 and Theorem 3.8 that the result obviously holds.

It is obvious that Corollary 3.10 coincides with [7, Theorem 3.2] if $F$ is a vectorvalued mapping and the closedness of $S_{w}(\cdot)$ is not considered.

Corollary 3.10 includes and improves [8, Theorem 3.1] and [11, Theorem 4.2 and Corollary 5.1], respectively, because the uniform compactness of $A$ is not required, see also [7, Corollaries 3.1 and 3.2].

\section{References}

[1] L. Q. Anh and P. Q. Khanh, 'Semicontinuity of the solution set of parametric multivalued vector quasiequilibrium problems', J. Math. Anal. Appl. 294 (2004), 699-711.

[2] $\quad$ 'On the stability of the solution sets of general multivalued vector quasiequilibrium problems', J. Optim. Theory Appl. 135 (2007), 271-284.

[3] J. P. Aubin and I. Ekeland, Applied Nonlinear Analysis (John Wiley and Sons, New York, 1984).

[4] C. Berge, Topological Spaces (Oliver and Boyd, London, 1963).

[5] G. Y. Chen, X. X. Huang and X. Q. Yang, Vector Optimization: Set-valued and Variational Analysis (Springer, Berlin, 2005).

[6] C. R. Chen and S. J. Li, 'Semicontinuity of the solution set map to a set-valued weak vector variational inequality', J. Ind. Manag. Optim. 3 (2007), 519-528.

[7] C. R. Chen, S. J. Li and K. L. Teo, 'Solution semicontinuity of parametric generalized vector equilibrium problems', J. Global Optim. 45 (2009), 309-318. 
[8] Y. H. Cheng and D. L. Zhu, 'Global stability results for the weak vector variational inequality', J. Global Optim. 32 (2005), 543-550.

[9] F. Ferro, 'A minimax theorem for vector-valued functions', J. Optim. Theory Appl. 60 (1989), 19-31.

[10] F. Giannessi (ed.), Vector Variational Inequalities and Vector Equilibria: Mathematical Theories (Kluwer, Dordrecht, 2000).

[11] X. H. Gong, 'Continuity of the solution set to parametric weak vector equilibrium problems', J. Optim. Theory Appl. 139 (2008), 35-46.

[12] X. H. Gong and J. C. Yao, 'Lower semicontinuity of the set of efficient solutions for generalized systems', J. Optim. Theory Appl. 138 (2008), 197-205.

[13] N. J. Huang, J. Li and H. B. Thompson, 'Stability for parametric implicit vector equilibrium problems', Math. Comput. Modelling 43 (2006), 1267-1274.

[14] J. Jahn, Vector Optimization-Theory, Applications and Extensions (Springer, Berlin, 2004).

[15] P. Q. Khanh and L. M. Luu, 'Upper semicontinuity of the solution set to parametric vector quasivariational inequalities', J. Global Optim. 32 (2005), 569-580.

[16] K. Kimura and J. C. Yao, 'Semicontinuity of solution mappings of parametric generalized vector equilibrium problems', J. Optim. Theory Appl. 138 (2008), 429-443.

[17] _ ' 'Sensitivity analysis of solution mappings of parametric vector quasi-equilibrium problems', J. Global Optim. 41 (2008), 187-202.

[18] S. J. Li and C. R. Chen, 'Stability of weak vector variational inequality', Nonlinear Anal. 70 (2009), 1528-1535.

[19] S. J. Li, G. Y. Chen and K. L. Teo, 'On the stability of generalized vector quasivariational inequality problems', J. Optim. Theory Appl. 113 (2002), 283-295.

[20] S. J. Li and Z. M. Fang, 'On the stability of a dual weak vector variational inequality problem', J. Ind. Manag. Optim. 4 (2008), 155-165.

[21] J. Li and N. J. Huang, 'Implicit vector equilibrium problems via nonlinear scalarization', Bull. Austral. Math. Soc. 72 (2005), 161-172.

[22] W. Song, 'Vector equilibrium problems with set-valued mappings', in: Vector Variational Inequalities and Vector Equilibria: Mathematical Theories (ed. F. Giannessi) (Kluwer, Dordrecht, 2000), pp. 403-422.

SHENG-JIE LI, College of Mathematics and Science, Chongqing University, Chongqing, 400030, PR China e-mail: lisj@cqu.edu.cn

HUI-MIN LIU, College of Mathematics and Science, Chongqing University, Chongqing, 400030, PR China e-mail:1hmmath@163.com

CHUN-RONG CHEN, College of Mathematics and Science, Chongqing University, Chongqing, 400030, PR China e-mail: chencr1981@163.com 